\title{
1
}

\section{The Sound of Coughing Pigs}

In winter 20I5, a door unexpectedly opened on a group of pigs. Where a moment before the room had been filled with the sound of grunts, squeals, and squeaks, the author and his wife were greeted by an expectant silence as sixty healthylooking pigs turned toward the door, sat down on their behinds-and coughed. The surprisingly human sound of sixty coughing pigs has stayed with us to this day. For the purposes of this book, the sound is doubly significant because these coughing pigs had not received any antibiotics. Instead, they were part of an experiment by the pig farmer, who was giving us an impromptu two-hour tour of the farm's facilities.

Having heard of my research, the farmer insisted that I get an inside look at how conventional agriculture really works. The family farm specializes in fattening piglets and selling them for slaughter. While its capacity of 4,000 pigs is small compared to US concentrated animal feeding operations, the farm we were touring is a fairly typical example of pig production in northern Europe. One of only two surviving farms in the village, it had formerly also produced crops, raised cattle, and bred horses. However, following the 1960 , it had heeded the maxim of "get big or get out" and specialized in pig production. While it still grows cereals to feed its pigs, the farm's cattle have long since gone and horses are now mostly kept as lodgers paid for by wealthy urbanites. As part of a fine-tuned just-in-time production system, the farmer starts the day by checking international commodity prices on the Chicago Board of Trade's website. If the price is right, pigs are bought and sold with a click. Despite 
ingrained agricultural pessimism about market outlooks, business is going well. The farmer is a well-respected member of the local community and has just built another high-tech pig sty. And yet, the farm's future seems uncertain.

One of our farmer's main concerns is the increasing political focus on husbandry practices in conventional agriculture. Getting planning permission for the new sty was challenging enough because of local complaints about the smell. However, in an accurate premonition, the farmer is most concerned about mounting pressure on European Union (EU) regulators to restrict routine antibiotic use on farms. And this is where we come back to our coughing pigs: caught between competing expert narratives, the coughing pigs are the farmer's own DIY experiment to gauge whether antibiotics are really necessary for the farm's mode of production. It seems that they are. Although no costly disease diagnosis was made, the other piglets reaching the farm on the same lorry received antibiotics prophylactically and did not fall ill. In a business where the difference between profit and loss is decided by the length of time and the amount of feed it takes to produce an animal, coughing pigs are a problem. So what is the farmer to do? Sacrifice a business model that has worked for more than forty years or support lobbying efforts to delay the implementation of stricter antibiotic regulations? This was the dilemma the pig farmer posed to us. What follows is the history of how this antibiotic dilemma came about.

\section{The Antibiotic Dilemma}

Antibiotics are part of a wider family of antimicrobial drugs with activity against a variety of microorganisms, including bacteria, viruses, fungi, and other eukaryotic parasites. Revolutionizing the medical marketplace from the early twentieth century onwards, modern antimicrobials comprise synthetic antimicrobials (e.g., sulfonamides), biological antibiotics-substances produced by microorganisms to act against other microorganisms (e.g., penicillin), and semisynthetic or modified biological antimicrobials (e.g., methicillin). ${ }^{1}$ In public discourse, the terms antibiotic and antimicrobial are frequently conflated. For the sake of simplicity, this book uses the most well-known term: antibiotic.

The modern prominence of antibiotics is hard to exaggerate. In schools, children learn the story of Alexander Fleming's 1928 discovery of the antibacterial qualities of the Penicillium notatum mold, museums feature exhibitions on "yellow magic," and patients and doctors routinely take and prescribe antibiotics for various ailments. So common and important have antibiotics become that recent books even talk of an "antibiotic era" in human medicine from the 1930s onward. What is, however, often forgotten is that antibiotics have also come to play a significant role in food production. In fact, more than 50 percent of global antibiotic production is not destined for human use. ${ }^{3}$ 
The origins of non-human antibiotic use lie in the interwar period. Starting during this time, dramatic changes began to transform livestock production. Over the following decades, sizes and animal concentrations grew rapidly while new breeds and production systems changed the biological rhythms of livestock production. Although there were significant variations between different sectors and regions, growing numbers of animals disappeared into confined high-input housing systems or mixed indoor-outdoor systems. Breeding programs and fierce competition resulted in the dominance of a small number of animal breeds that were particularly efficient at converting feed into meat. Meanwhile, concentration processes increased both the productivity of animal husbandry and the investments necessary to survive oversaturated markets. In a process known as vertical integration, many producers now contract or work directly for larger corporations, which often control not only animal production but also feed production, slaughtering, and processing. ${ }^{4}$ Changes were particularly impressive in the poultry industry. Whereas a meat-producing broiler chicken took II2 days to reach an average market weight of 2.8 pounds in 1935, it only needed 47 days to reach a live market weight of 4.7 pounds in 1995. ${ }^{5}$ Chickens' bodies changed accordingly. Starting in the 1950s, heavier broiler chickens began to replace older varieties like the Rhode Island Red. ${ }^{6}$ Since then, poultry meat has become a cheap and popular food with a small number of companies dominating international production. ${ }^{7}$ Pig and cattle production have also become more concentrated-although the process was often more fragmented and confinement slower to develop. ${ }^{8}$ In the twenty-first century, the intensive (confined and concentrated) and industrial (integrated) production of animals is fast becoming the global norm.'

One of the most formidable obstacles faced by expanding animal production is infectious disease. Prior to the interwar period, farmers had already attempted to increase herd densities. However, despite the use of antibacterial compounds like organoarsenics, infectious disease remained a serious threat. This situation changed during the 1940s. Within a decade, cheap antibiotics became routine components of the agricultural fight against bacterial infection. ${ }^{10}$ Farmers soon found that antibiotics could also be used prophylactically to prevent infections from spreading in the first place. A significant third factor contributing to agriculture's antibiotic adoption was that even small doses of some antibiotics - if fed regularly_allegedly enabled animals to metabolize feedstuffs more efficiently. The mechanics behind the so-called antibiotic growth effect remain unclear. While postwar researchers believed that antibiotics optimized the microbial flora in animals digestive systems, ${ }^{11}$ contemporary theories posit $(\mathrm{I})$ that by inhibiting bacterial digestion, antibiotics maximize the amount of available sugar, (2) that feeding antibiotics favors vitaminproducing bacteria and combats toxin-producing bacteria, and (3) that antibiotics favorably change the acidity of animals' stomachs. ${ }^{12}$ 
Although the past seven decades have seen consistent controversies over the mechanisms, extent, and very existence of the antibiotic growth effect, ${ }^{13}$ antibiotics' tripartite function of combating and preventing infection and saving feedstuffs was a winning combination. Alongside other similarly important interventions like new vaccines, improved housing, nutrition, and breeds, ${ }^{14}$ antibiotics aided a significant reduction of animal mortality. Whereas mortality in US broiler production was Io percent in 1945 , it sank to 8 percent in 1950 , 6 percent in 1960, and 4.8 percent in 2015 . Meanwhile, feed efficacy improved from 4 pounds of feed consumed to produce one pound of meat in 1945 to 2.5 pounds in 1960 and $\mathrm{I} .82$ pounds in $2018 .{ }^{15}$ Then as now, the boundaries between therapeutic, prophylactic, and growth promotional antibiotic use frequently blurred. Antibiotics also entered other areas of food production. In addition to a brief career as food preservatives, they are still used to combat bacterial infections of crops and fruit and to protect bees. ${ }^{16}$ While it is important to note that agricultural industrialization would have occurred with or without their discovery, ${ }^{17}$ cheap antibiotics thus greatly facilitated the monoculture-like concentration of organisms and the substitution of human labor in modern livestock production. Over time, antibiotics' extensive use created significant infrastructure-like physical and cultural dependencies in global food production. ${ }^{18}$

While antibiotic infrastructures continue to influence contemporary husbandry and disease management systems, the resulting chemical cornucopia has also come at a price. Agricultural antibiotics face three different strands of criticism. First, according to some critics, antibiotics have enabled a neglect of animal welfare and allow inhumane factory farms to profit from animals' suffering. Second, many consumers and health authorities are also concerned about drug residues in food, water, and the environment. Some antibiotics are allergenic and can either sensitize individuals or trigger existing antibiotic allergies. ${ }^{19}$ Allergic reactions can range from stomach irritations to the eruption of painful hives on the skin. In the worst case, allergies can trigger a life-threatening anaphylactic shock when antibiotics are administered in higher doses. A third and increasingly vocal group of critics focuses on antibiotics' selection for antimicrobial resistance (AMR). ${ }^{20}$

Although agriculture's exact contribution to the global AMR burden remains contested, bacteria's increasing ability to "resist" antibiotics is now widely held to be one of the most pressing global health challenges of the twentyfirst century. The causes of AMR are complex. Bacteria can be either intrinsically resistant to an antibiotics or acquire resistance to it. In the case of intrinsic resistance, the natural characteristics of a bacterium's biology (e.g., cell wall or metabolism) can make it "immune" to certain antibiotics. For example, the iconic penicillin $\mathrm{G}$ is ineffective against most gram-negative bacteria, which possess double cell walls. Other bacteria may also already "naturally" possess the mechanisms with which to "resist" antibiotics, such as enzymes that deactivate 

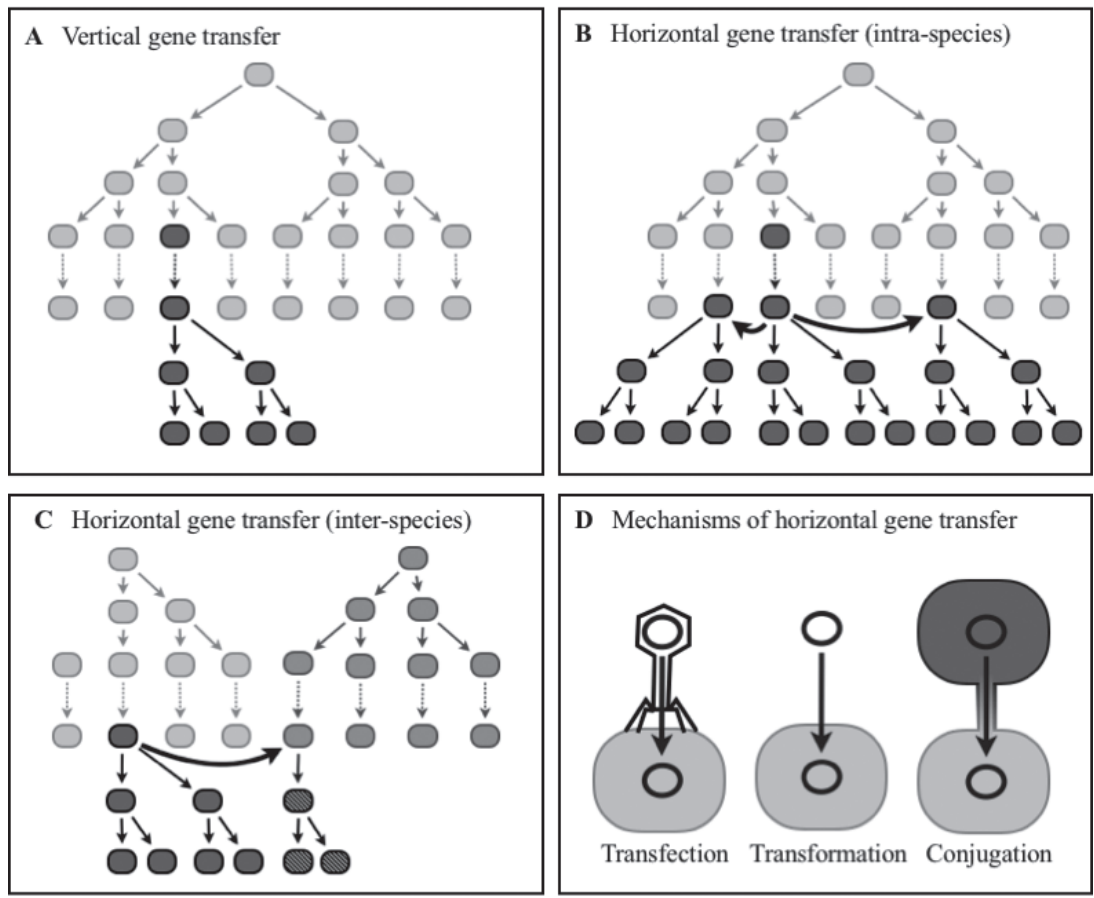

D Mechanisms of horizontal gene transfer
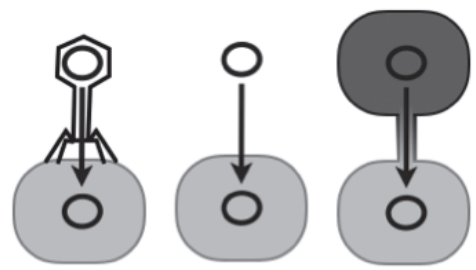

Transfection Transformation Conjugation

FIGURE A.1 Modes and mechanisms of AMR transfer.

antibiotics, the ability to modify antibiotic target sites, or efflux pumps with which to pump antibiotics out of the bacterial cell. By contrast, acquired resistance arises from spontaneous bacterial mutations and the acquisition of resistance-conferring mobile genes. Increased tolerance to an antibiotic will give a bacterium an evolutionary advantage over its peers the next time it is exposed to the respective antibiotic or to substances with similar effects (co-selection). ${ }^{21}$

Intrinsic and acquired resistance can pass from one bacteria generation to the next (vertical gene transfer). However, bacteria also possess the remarkable ability to "communicate" information on how to resist antibiotics among each other in a process called horizontal gene transfer. Whereas mutations bring new AMR genes into the world, horizontal gene transfer is the major force spreading these genes across the globe. Horizontal gene transfer can either occur as a result of transduction, during which resistance genes (R-factors) are inserted into the bacterial genome by viral bacteriophages; transformation, during which bacteria absorb free-floating resistance-encoding DNA sequences; and as a result of conjugation ("bacterial sex"), during which bacteria exchange small circular DNA strands called plasmids, which encode R-factors. ${ }^{22}$

It is difficult to exaggerate the implications of horizontal gene transfer. Because genetic information can be exchanged between bacteria of different 
species, an antibiotic-resistant yet innocuous Escherichia coli (E. coli) bacterium can pass on its resistance to a pathogenic Salmonella bacterium. Significantly, it is often not just resistance against one but against several antibiotics that is transferred en bloque. This en bloque transfer occurs because resistance genes tend to be stockpiled in mobile regions of the bacterial genome that are more easily transferred to other bacteria - the mobilome-such as plasmids and integrons. Any bacterium receiving such a genomic island via horizontal gene transfer can immediately resist multiple antibiotics. Exposing this bacterium to one of these antibiotics or related substances will automatically co-select for all of the other resistance information encoded on the genomic island. ${ }^{23}$ Even sublethal concentrations of antibiotics, such as diluted antibiotics in rivers or in animal feeds and medicines, can select for clinically relevant resistance genes. $^{24}$

Once established, resistant organisms and genes routinely cross geographic and species borders. With approximately 60 percent of all infectious diseases (and 75 percent of emerging infectious diseases) affecting humans shared by other vertebrate animals, the selection of resistant pathogens in one population can have serious consequences for the other. ${ }^{25}$ This is not only true for established zoonotic pathogens but also for seemingly harmless bacteria. In the case of livestock-associated methicillin-resistant Staphylococcus aureus (CC398 MRSA), researchers found that the strain had originated as an antibiotic susceptible human Staphylococcus aureus strain that had jumped to livestock, where it had acquired resistance to methicillin and tetracycline (probably as a result of agricultural antibiotic use) before reinfecting humans in contact with farm animals. ${ }^{26}$ Horizontal gene transfer means that a similar ecological blurring of AMR risks is also occurring because of the spread of AMR genes selected in one species and geographic environment to another.

Decades of continuous antibiotic use have led to a significant enrichment and selection for AMR in the global microbiome. It is uncertain whether there is a way back from this Anthropocene of the cell. ${ }^{27}$ Although the higher energy cost of maintaining AMR means that some bacteria may regain sensitivity once selection pressure is decreased, others may not. The case of the mobilized colistin resistance-I (mcr-I) allele serves as a warning. In November 2015, Lancet Infectious Diseases reported that resistance against colistin, a member of the old antibiotic class of the polymyxins, had been detected in E. coli samples from Chinese pigs. Polymyxins were commonly used in Chinese agriculture but also serve as last-resort antibiotics in Western hospitals. Chromosomal polymyxin resistance via point mutations was well known and had relatively high fitness costs. What was alarming about the 2015 resistance detection was that the $m c r-I$ allele was encoded on a plasmid (i.e., prone to horizontal gene transfer) and associated with significantly lower fitness costs. Because polymyxins were rarely used in Chinese hospitals, AMR in local human populations had probably 
arisen in agriculture; $m c r-I$ spread rapidly. ${ }^{28} \mathrm{Within}$ a year, researchers had detected it in human and animal samples from five continents. Although plasmids containing mcr-I have, at the time of writing, failed to accumulate resistance to carbapenems, the other group of antibiotics used to treat resistant Enterobacteriaceae, this is likely only a matter of time. ${ }^{29}$ The case of $m c r-I$ highlights the potential of untreatable pandemic strains emerging as a result of local antibiotic exposure in humans, animals, or the environment.

\section{Farmyard Realities}

Faced with surging AMR and no new antibiotic classes since the 1980 , a growing number of politicians, experts, and consumers agree that the combined price of AMR, residues, and inadequate welfare outweighs the productivity benefits conferred by agricultural antibiotic use. Others disagree. Back on the farm, our farmer's answer is mixed. In the case of residue and welfare allegations, the farmer denies that there are serious problems. Although individual black sheep may ignore guidelines, strict European testing and new legislation have solved previous problems. The farmer is more defensive when it comes to AMR: Yes, AMR is a significant problem. But who is to say that agricultural antibiotics are responsible for it? Aren't patients and doctors more to blame for antibiotic overuse? Don't consumers drive agricultural antibiotic use by demanding cheap meat? And why should the farmer stop using antibiotics if foreign competitors continue to use them?

The farmers' opinions are legitimate and should be taken seriously. They also reflect more than seventy-five years of disputes over agricultural antibiotic regulation and increasingly fragmented notions of antibiotic risk. Understanding the factors driving this fragmentation of perceptions and resulting impacts on antibiotic regulation is the central aim of this book. Breaking with previous historiography's emphasis on human antibiotic use, it provides a unifying lens through which to analyze the often contradictory actions and perceptions of multiple actors and groups. What emerges is a familiar story of spreading dependencies and narrowly conceived regulatory solutions. Perhaps more importantly, it is also a story of how one group of substances acquired multiple meanings in different communities. This second story not only complicates moralistic accounts of "Big Ag" or "Big Pharma," it also serves as a cautionary tale about short-term thinking when it comes to current drug licensing and antibiotic stewardship. ${ }^{30}$

Two observations on the nature of risk and group formation help the book tackle its topic. The first observation is that differing risk cultures (epistemes) have played a crucial role in allowing global antibiotic use and AMR to rise to current levels. Because risk epistemes are difficult to reconstruct by looking at one nation in isolation, the book compares the development of antibiotic risk 
epistemes in the United States and the United Kingdom. Both nations were key to structuring the antibiotic infrastructure underpinning modern food production. However, the comparative analysis shows that distinct cultural priorities, agricultural infrastructures, and regulatory path dependencies produced very different risk epistemes. The book's second central observation is that each national risk episteme was strongly influenced by how antibiotic risk was staged in three overlapping social spheres: the general public, the agricultural community, and the regulatory community. Communities in each sphere developed their own understanding of antibiotics' risks and benefits. Over time, diverging understandings fostered the formation of rival groups and triggered increasingly fierce clashes over how agricultural antibiotics should be viewed, used, and regulated. Clashes occurred both within and between spheres. The wider national risk episteme was a continuously evolving reflection of these domestic clashes.

The book's twin focus on risk epistemes and communicative spheres is inspired by sociologists Ulrich Beck and Niklas Luhmann. In his seminal Risikogesellschaft (1986), Ulrich Beck highlighted the importance of risk for driving societal action. According to Beck, risk is the probability-based anticipation of catastrophe but not the catastrophe itself. Societies and individuals constantly strive to avoid predicted risks. Because everything can theoretically be interpreted as a risk, there is a constant struggle over whether a risk is real, negligible, or urgent. ${ }^{31}$ Risk's infinite nature also means that no definitive expertise is possible. In the virtual world of risk, "no one is an expert or everyone is an expert." ${ }^{32}$ As a consequence, the "objectivity of a risk is the product of its [cultural] perception and [social/objective] staging." ${ }^{\prime 33}$ Within every society, existing inequalities mean that certain groups will be more successful at staging their version of risk and distributing resulting burdens than others. Often, the poorest and weakest end up bearing the greatest risk. Understanding how riskbased group identities emerge and interact is crucial if one wants to unravel the patchwork of national and international regulations that has emerged around antibiotic use. It is here that the communicative sociology of Niklas Luhmann can serve as a useful tool. According to Luhmann, every society comprises multitudes of smaller subsystems with relatively autonomous modes of communication: the smaller the group, the more distinct its communication and attitudes. Each subsystem or sphere attempts to frame or stage its version of reality for other spheres. A society's values, identities, and risk definitions are the contingent result of this potpourri of competing worldviews. ${ }^{34}$

As the anecdotal example of our pig farmer and recent social sciences research show, perceptions of antibiotic risk have certainly begun to differ between farmers and the wider public as well as between different countries-even experts' risk heuristics have been found to be affective and culturally biased. ${ }^{35}$ These findings should not surprise us. Over the past three decades, science and 
technology studies (STS) research has revealed the rootedness of risk in distinct—often national—contexts. Sheila Jasanoff has described how cultural values, legal systems, and differing modes of knowledge production have created unique "civic epistemologies." ${ }^{36}$ Historians have also explored who gets to decide whether risks are relevant, can be regulated, and should be monitored. In this context, Alexander Schwerin has highlighted the role of national "risk epistemes" - the complex nexus of political interests, expert epistemologies, institutional path dependencies, and technological heuristics-in deciding over the perception and management of environmental and technological hazards. ${ }^{37}$

Emphasizing the role of risk evaluation and management is important. In contrast to an unavoidable calamity, risk is both a hazard and an opportunity. Historically, formal risk definitions have played a significant role in transforming hazardous technologies from unpredictable dangers into calculable and thus manageable entities. ${ }^{38}$ Phenomena that can be predicted can be accounted for and integrated into capitalist modes of redistribution and commodification. In the context of chemical and pharmaceutical regulation, numerous historians and STS scholars have pointed to the role of threshold models and boundary values in allowing hazardous substances to permeate workspaces, environments, and human bodies. In contrast to a zero-tolerance attitude toward risk, exposure to chemicals, radiation, and other hazards is deemed acceptable if it stays below a defined level. Large parts of industrial production now depend on the normalization of risk by a specialized regulatory branch of science. ${ }^{39}$ For those who are unwilling to accept this normalization of risk, a lucrative market offers ways to further reduce exposure via insurance, "pure" food, or "safe" housing. However, not everybody can afford to opt out of normal risk. In most countries, risk exposure varies significantly along familiar socioeconomic divides. ${ }^{40}$

What is generally accepted as normal risk can also shift. Tolerance of technologies is often linked to historically fluid cultural notions of purity and naturalness. ${ }^{41}$ According to Sheila Jasanoff: "The demarcation between the natural and the unnatural in any society is not given in advance but is crafted through situated, culturally specific forms of boundary work." ${ }^{42}$ For regulators, the shifting results of public, industrial, and scientific boundary work pose a constant challenge: What if some experts say that the theoretical risk of a new technology is too great for it to be licensed but others disagree? Should a potentially beneficial technology be licensed even if prevalent cultural sentiment is against it? Should one wait until concrete proof of harm arises or should one listen to societal "gut instincts" and enforce precautionary bans? What if a technology is already on the market and new evidence challenges previous safety models? Over time, two contested philosophies have developed to address this epistemic challenge. One philosophy holds that concrete evaluations of proven 
costs and benefits should decide over technological regulation. The other philosophy holds that precautionary bans are justifiable if there is strong reason to suspect harm-even without conclusive evidence of it having occurred. ${ }^{43}$

The long conflict about agricultural antibiotics takes us right to the heart of the competitive staging of risk, its distribution, and the intricacies of precautionary and cost-benefit regulation. From the 1940s onward, societies had to decide which of antibiotics' benefits to enjoy at the cost of which risk. Meanwhile, changing conceptions of food safety and evolving AMR science repeatedly led to redefinitions of antibiotics' "true risk." Caught between agro-industrial interests and vocal medical, consumer, and animal welfare activists, regulators struggled to define boundary values for safe agricultural antibiotic use. One person's meat was another person's poison. Over time, different risk epistemes led to divergent regulation: reacting to industry pressure and public concerns, US regulators focused on monitoring food and milk for antibiotic residues. Meanwhile, new surveillance technologies and popular concerns made British regulators prioritize AMR over residue hazards. In 1969, the British Swann report recommended precautionary bans of low-dosed growth promoter feeds containing medically relevant antibiotics. Swann-inspired growth promoter bans were subsequently adopted in Britain and the European Economic Community (EEC) but not in the United States, where regulators continued to focus on preventing residues and favored cost-benefit evaluations. In recent decades, the regulatory gap has widened with the EU expanding antibiotic growth promoter bans between 1997 and 2006 and announcing plans to ban prophylactic and forms of metaphylactic (control treatment of animal groups) antibiotic use by 2022 in late $2018 .^{44}$ Although the effects of bans on AMR remain contested, EU agricultural antibiotic sales roughly stagnated between 2011 and 20I4 with approximately 9,909 tons consumed in 2014 before falling by about 20 percent to 7,860 tons in $2016 .{ }^{45}$ By contrast, the United States did not enact full statutory bans. While sales increased by 22 percent to 15,576 tons between 2009 and 2015, voluntary feed restrictions, industry reforms, regulatory threats, and changing consumer preferences have, however, led to a significant drop of consumption by about 30 percent to I0,933 tons between 2015 and $2017 .^{46}$

This book's four-part structure reflects its transnational scope and the importance of the three domestic spheres-public, agricultural, and regulatory-in shaping national risk epistemes. Each part studies a distinct phase of British or US antibiotic history and is divided into three chapters. Of these, one focuses on the public, one on the agricultural, and one on the regulatory perception and management of agricultural antibiotics. Inspired by Michelle Mart's analysis of US attitudes toward pesticides, each chapter explores dominant themes and mutually reinforcing discourses on antibiotics. ${ }^{47}$ 
Analyzed sources include national and regional newspapers, consumer and activist publications, fashion and lifestyle magazines, cookery books, organic and conventional farming magazines, agricultural and veterinary manuals, industry publications, and archival material from regulatory agencies. However, there remain limits to what these sources can tell us. Many of them are elite and were written about rather than by actors themselves. Meanwhile, media reporting on antibiotics was often monolithic and conservative in its framing of mainstream opinion. There is also no guarantee that information in handbooks, newspapers, or official publications was taken seriously by producers or consumers. Finally, the sheer wealth of material means that it is impossible to cover all of the nuances of antibiotic opinion. This means that depictions of consumers, regulators, and farmers will often remain ideal types-a fact exacerbated by the book's transnational comparison, which to a certain degree depends on generalizable categories. However, these limitations should not deter curiosity or research. Although their coverage remains eclectic, keywordsearchable databases now give historians access to an unprecedented wealth of material on actors' lives and opinions. The digital repositories informing this book have been chosen to reflect as many political and regional perspectives as possible. While no single source group can fully reconstruct actors' opinions and actions, grouping as many as possible together provides a more detailedif still grainy-picture.

The book's four parts are ordered chronologically. Following a brief introduction to antibiotics and AMR, part I studies the emergence of agricultural antibiotic use in the United States. Chapter 2 describes the initial public optimism about agricultural antibiotics before analyzing the effects of 1950 s concerns about chemical additives, carcinogens, and food purity on substances' image. The chapter also highlights the divide between risk perceptions of AMR in medical and agricultural settings. Chapter 3 shows that antibiotics quickly acquired infrastructural relevance within US agriculture and were seamlessly incorporated into existing feed industry marketing channels. Focusing on risk perceptions among conventional and organic farmers, it highlights that US farmers knew little about the new miracle drugs' effects or potential hazards. Chapter 4 then describes the challenges US officials faced when regulating antibiotics. It shows that agricultural antibiotics' risks were initially deemed negligible by a regulatory matrix that focused on "classic" toxic and carcinogenic hazards. Lacking resources and a coherent policy framework, regulators later struggled to enforce drug compliance and respond to AMR warnings.

Part 2 focuses on Britain. Chapter 5 reconstructs how British public perceptions of antibiotic risk differed from those in the United States. During the I960s, traditional concerns about animal welfare and new warnings about infectious resistance on factory farms created a powerful alliance for AMR-focused 
reform. Chapter 6 studies antibiotics' hesitant adoption by Britain's diverse agricultural community. It discusses the importance of close corporatist ties between the National Farmer Union (NFU) and government officials in overcoming initial skepticism regarding antibiotics and ensuring that later reforms did not harm farmers. Chapter 7 reconstructs how British officials first licensed antibiotics and then reviewed their use three times during the 1960 s. Although their demands were repeatedly watered down, public health experts' use of a technology called bacteriophage typing to track the spread of resistant organisms played a decisive role in forcing officials to implement precautionary yet narrow restrictions of antibiotic feeds as a result of the 1969 Swann report.

Part 3 returns to the United States. Chapter 8 asks why US perceptions of agricultural antibiotics remained relatively unaffected by European AMR concerns. Between the I970s and the mid-1990s, public opinion on antibiotic stewardship remained divided. This division was caused by ongoing residue fears, concerns about "stagflation," and the increasingly partisan nature of environmentalist politics. Chapter 9 highlights how US farmers struggled to come to terms with non-agricultural criticism of antibiotic use during a time of increasing economic pressure. It shows how hostility toward government intervention and a seeming lack of alternative production methods made most conventional farmers support lobbyists' campaigns against antibiotic restrictions. Although the mid-r980s saw organic intensification and financial pressure facilitate a market-driven conciliation with conventional agriculture, it did not trigger a wider reform of production methods. Chapter Io analyzes US officials' reaction to new risk scenarios involving horizontal gene transfer. It shows that officials were divided in their assessment of precautionary European bans and underestimated industry opposition to antibiotic reform. After failing to restrict agricultural antibiotic use four times during the 1970s, government officials reverted to earlier policies of nonstatutory reform.

Part 4 covers the era following the implementation of the Swann report in Britain. Chapter II reconstructs the 1970 s fragmentation of public pressure for antibiotic reform, the re-emergence of concerns about agricultural AMR selection around 1980, and the 1996 bovine spongiform encephalopathy (BSE) crisis's role in reigniting national reform campaigns. Chapter I2 studies the limited impact of initial antibiotic restrictions on British farming. After a brief reduction during the early 1970 s, agricultural antibiotic use soon recovered and continued to increase until the late 1990s. Similar to the United States, most British farmers continued to see antibiotics as an important component of modern production and risk management. It was only when corporatist decisionmaking began to fracture during the 1980 os that AMR and residue hazards were discussed more extensively. Following the 1996 BSE crisis, agricultural opposition to further growth promoter bans was limited. Chapter 13 asks why the 
Swann report failed to curb either antibiotic use or AMR. It emphasizes the importance of interministerial rivalry and corporatist ties in watering down many proposals of the original report. With British efforts stagnating, European integration played a decisive role in reinvigorating British antibiotic reform from the rig8os onward.

Returning to our farmer and the coughing pigs, chapter 14 recapitulates the tumultuous history of agricultural antibiotics' perception, use, and regulation. It asks whether this history can provide insights for current regulators. The chapter tells four cautionary stories about narrow reforms, infrastructural entanglements, epistemic fragmentation, and short-termism before arguing for a new policy emphasis on microbial resilience. 
